\title{
Doped Mesoporous Carbons Derived from Transition Metal Iron and Chitosan as Efficient Non-Precious Cathode Catalysts for Oxygen Reduction Reaction in Alkaline Electrolyte
}

\author{
Fang Dong ${ }^{1}$, Enguang Zhang ${ }^{1}$, Qiaowei Tang ${ }^{1}$, Qinping Guo ${ }^{2^{*}}$ and Jinli Qiao ${ }^{1^{*}}$ \\ ${ }^{1}$ College of Environmental Science and Engineering, Donghua Universtiy, \\ 2999 Ren'min North Road, Shanghai 201620, China \\ ${ }^{2}$ College of Material Science and Engineering, Taiyuan University of Technology, \\ Taiyuan 030024, Shanxi, China \\ E-mail address: *qiaojl@dhu.edu.cn, guoqingping6502@163.com
}

\begin{abstract}
Keywords: Oxygen reduction reaction, mesoporous carbon materials, transition metal iron, chitosan, alkaline electrolyte
\end{abstract}

Abstract. In this work, a series of mesoporous carbonaceous materials (MCs), which are simultaneously doped with $\mathrm{N}$ and Fe-containing species, were prepared by polymerization of potassium hexacyanoferrate-mediated chitosan on $\mathrm{SiO}_{2}$ nanospheres. The $\mathrm{SiO}_{2}$ template was carbonized and etched with $\mathrm{NaOH}$. The electrochemical properties of the $\mathrm{MCs}$ as nonprecious-metal electrocatalysts for oxygen reduction reaction (ORR) are characterized, which was performed using cyclic voltammetry $(\mathrm{CV})$ and linear sweep voltammetry (LSV) employing a rotating disk electrode (RDE). The results show that chitosan with well-dispersed $\mathrm{K}_{4} \mathrm{Fe}(\mathrm{CN})_{6}$ nanoparticles prepared by annealing at $800^{\circ} \mathrm{C}$ (CTS-Fe-800) exhibits the highest catalytic performance in terms of ORR among the catalysts studied, where the onset potential at $0.91 \mathrm{~V}$, half-wave potential at $0.82 \mathrm{~V}$ and average transferred electron number of 3.49 , were achieved in alkaline media.

\section{Introduction}

Faced with the increasingly serious energy crisis, fuel cells, as a clean and efficient power source, have become the most promising energy conversion devices and attracted significant attention during the last decades. Oxygen reduction reaction (ORR) at the cathode of fuel cells plays a decisive role in determination of the performance, and electrocatalysts with high-performance ORR are essential for practical applications. Pt-based materials are widely recognized as superior electrocatalysts for ORR [1], however, they are hindering this technology's commercialization because of their expensive and scarce nature [2]. Therefore, overcoming the challenge is undoubtedly of particular importance. A promising approach to solve this problem is to introduce non-noble metals such as Fe, Co [3] or their compounds into the nitrogen-doped carbon material since the N-doped carbon can exhibit good electrocatalytic activities to the ORR [4] and incorporation of a metal can significantly improve the catalytic efficiency [5].

Extensive efforts have been devoted to develop cheap and earth abundant alternative electrocatalysts [6]. In fact, certain types of N-containing transition metal catalysts have shown high activity for oxygen reduction in alkaline media [7, 8], however, compared to Pt-based catalysts for practical application in fuel cells, both the ORR activity and stability of these catalysts have to be significantly improved.

It is believed that the high surface area, high porosity, and appropriate pore structure of the catalyst generally leads to a high catalytic activity for ORR [9]. In this paper, we choose the template method for the synthesis of transition metal Fe and $\mathrm{N}$ co-doped mesoporous carbon as 
non-noble metal catalysts. Nanoscale $\mathrm{SiO}_{2}$ was used as a sacrificial support to produce pores, while chitosan (CTS) was used as the source of both $\mathrm{N}$ and $\mathrm{C}$.

\section{Experimental}

Catalyst preparation. For obtaining the mesoporous carbon materials, the chitosan solution was prepared at first as follows: $5 \mathrm{~mL}$ glacial acetic acid was dissolved in $200 \mathrm{~mL}$ deionized water to get a $4 \mathrm{M} \mathrm{CH}_{3} \mathrm{COOH}$ solution. Then, $0.76 \mathrm{~g}$ chitosan was dissolved in the above solution under magnetic stirring to form a uniform solution.

The CTS-Fe catalyst was prepared by homogeneously dispersing potassium ferrocyanide (Sinopharm Chemical Reagent Co) and chitosan (Sinopharm Chemical Reagent Co) precursors onto the surface of $\mathrm{SiO}_{2}$ (Hangzhou Wanjing New Material Co, Ltd, $15 \pm 5 \mathrm{~nm}$ ). In a typical synthesis process, the pre-synthesized chitosan solution was added into a calculated amount of $\mathrm{SiO}_{2}$ solution (silica amount: $5 \mathrm{~g}$ ) under magnetic stirring. Then, a solution of $\mathrm{K}_{4} \mathrm{Fe}(\mathrm{CN})_{6}$ was added under stirring for 15 minutes. The resulting solution was further heated in $85^{\circ} \mathrm{C}$ water bath for 4 hours to mix sufficiently. After heated, the resulting viscous solution was filtration to obtain the light green solid $\mathrm{CTS}-\mathrm{Fe} / \mathrm{SiO}_{2}$, and then dried overnight at $85^{\circ} \mathrm{C}$. The solid $\left(\mathrm{CTS}-\mathrm{Fe} / \mathrm{SiO}_{2}\right)$ was ground to a fine powder in an agate mortar and pyrolyzed at 700,800 , and $900^{\circ} \mathrm{C}$, respectively, for 1 hour under a nitrogen atmosphere, with temperature ramp rates of $5^{\circ} \mathrm{C} / \mathrm{min}$. The $\mathrm{SiO}_{2}$ was leached out using excess amounts of $5 \mathrm{M}$ sodium hydroxide $(\mathrm{NaOH})$ for 48 hours and dried for overnight. Then the final catalyst samples were obtained and denoted as CTS-Fe-700, CTS-Fe-800, CTS-Fe-900, respectively.

Electrochemical testing. $5.0 \mathrm{mg}$ of CTS-Fe- $\mathrm{x}(\mathrm{x}=700,800,900)$ catalyst was dispersed in a mixed solution containing $800 \mu \mathrm{L}$ isopropyl alcohol (Sinopharm Chemical Reagent Co, Ltd) and $200 \mu \mathrm{L} 0.5 \%$ Nafion (diluted with methanol) to prepare the catalyst ink. Then $5 \mu \mathrm{L}$ of ink was applied onto a clean glassy carbon (GC) disk three times, with a sectional area of $0.2475 \mathrm{~cm}^{2}(5.6$ $\mathrm{mm}$ diameter). Then GC disk was ready to work after air-drying with the overall loading of $300 \mu \mathrm{g}$ $\mathrm{cm}^{-2}$. The current densities reported here all use the geometric area of the carbon electrode and all potentials were referred to a reversible hydrogen electrode (RHE).

Electrochemical analysis for the synthesized catalysts was performed by CV, LSV and RDE measurements using a CHI Electrochemical Station (Model 750b) and a conventional three electrode cell filled with $0.1 \mathrm{M} \mathrm{KOH}$ electrolyte saturated in $\mathrm{O}_{2}$ at room temperature. The measurements were carried out using RDE (Pine 5908 Triangle Drive Raleigh, NC21617) by CV and LSV at a slow scan rate of $50 \mathrm{mV} \mathrm{s}^{-1}$ and $5 \mathrm{mV} \mathrm{s}^{-1}$, respectively, at a rotation speed of 300,600 , 900, $1200,1600 \mathrm{rpm}$, respectively, to determine the Koutecky- Levich plots.

\section{Results and Discussion}

Cyclic voltammetry. CV curves were employed to compare the electrocatalytic activity of CTS-Fe-700, CTS-Fe-800, and CTS-Fe-900 for ORR in $\mathrm{N}_{2}$ - or $\mathrm{O}_{2}$-saturated $0.1 \mathrm{M} \mathrm{KOH}$ solution, as shown in Fig. 1. It can be seen that the CV curves of CTS-Fe-700, CTS-Fe-800, and CTS-Fe-900 are essentially featureless in the $\mathrm{N}_{2}$ saturated solution, whereas the three catalysts exhibit significant cathodic oxygen reduction peaks in the $\mathrm{O}_{2}$-saturated electrolyte. Compared with CTS-Fe-700 and CTS-Fe-900, CTS-Fe-800 displayed a more pronounced ORR peak, a more positive peak potential $(0.73 \mathrm{~V}$ vs. $0.71 \mathrm{~V}$ vs. $0.66 \mathrm{~V})$ and a higher corresponding current density $\left(-2.1 \mathrm{~mA} \mathrm{~cm}{ }^{-2}\right.$ vs. -2.6 $\mathrm{mA} \mathrm{cm}{ }^{-2}$ vs. $-4.1 \mathrm{~mA} \mathrm{~cm}^{-2}$ ). 


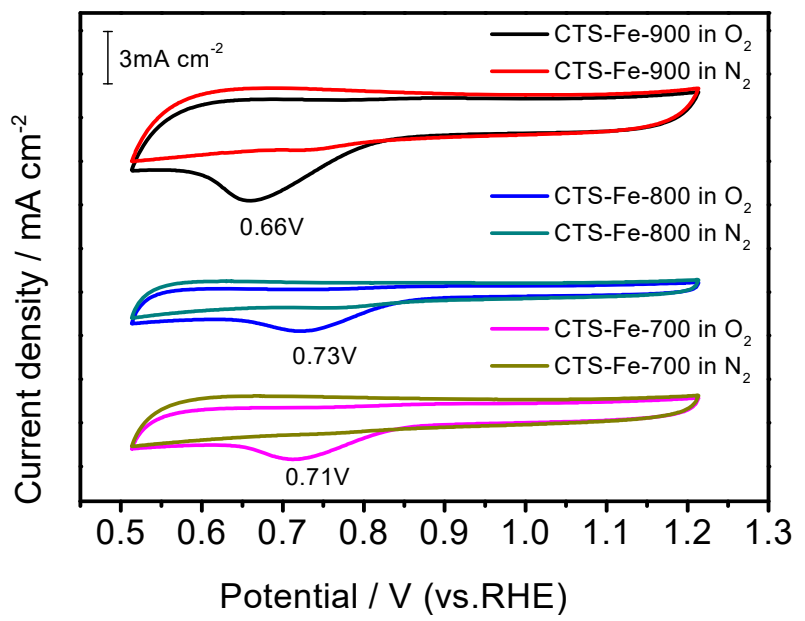

Fig.1 CV curves of CTS-Fe-700, CTS-Fe-800, CTS-Fe-900 in $\mathrm{N}_{2}$ - and $\mathrm{O}_{2}$-saturated $0.1 \mathrm{M}$ $\mathrm{KOH}$ solution (scan rate: $50 \mathrm{mV} \mathrm{s}^{-1}$ ). The overall loading of the mixed catalyst: $80 \mu \mathrm{g} \mathrm{cm}^{-2}$.

The results strongly indicate that the temperature have a significant effect on improving the ORR catalytic activity, and the catalyst annealed at $800^{\circ} \mathrm{C}$ has a higher performance towards ORR. Since the catalytic activity of pure oxidized iron species for ORR is low, the high activity of CTS-Fe-800 could be attributed to a synergetic effect between the doped $\mathrm{N}$ and $\mathrm{K}_{4} \mathrm{Fe}(\mathrm{CN})_{6}$.

RDE measurements. The effect of the pyrolysis temperature in the range of $700 \sim 900^{\circ} \mathrm{C}$ on the ORR activity of the CTS-Fe-x catalyst was also studied using RDE technique. The polarization curves in Fig. 2(a) show that the CTS-Fe-800 exhibits a higher ORR activity than CTS-Fe-700 and CTS-Fe-900 in terms of both onset potential and half-wave potential, as well as the current density over the whole potential range. These results imply that CTS-Fe-800 has a better catalytic activity for ORR than the other samples, confirming that the temperature is one of the main factors for its excellent electrocatalytic activity.

For clarifying the pathway and kinetic process of the ORR, polarization curves of the CTS-Fe-800 catalyst were recorded using the RDE technique at different rotation rates $(300,600$, 900,1200 and $1600 \mathrm{rpm}$ ) in $\mathrm{O}_{2}$-saturated solution with a scan rate of $5 \mathrm{mV} \mathrm{s}^{-1}$ (Fig. 2(b)). The curves show an increase in the catalytic current density with rotation rates because of the enhanced diffusion of electrolytes. Then the Koutecky-Levich plots for the ORR at different potential for CTS-Fe-800 can be obtained. The slope of the plots allows us to check the consistency with the theoretical values according to the Eq. 1:

$$
\frac{1}{j}=\frac{1}{j_{k}}+\frac{1}{j_{f}}+\frac{1}{j_{d}}
$$

Where $j$ is the measured current density at given potential, $j_{\mathrm{k}}$ is the activation controlled current density,jf is the effect of Nafion ionomer inside the catalyst layer on the measured current density, and $j_{d}$ is the $\mathrm{O}_{2}$ diffusion limiting current density. Due to the $j_{\text {f }}$ may be negotiable if the equivalent Nafion ionomer thickness is much small $\left(j_{f} \rightarrow \infty\right)$, therefore, its reciprocal can be negligible. In this case, Eq. 1 can be simplified to Eq. 2:

$$
\frac{1}{j}=\frac{1}{j_{k}}+\frac{1}{j_{d}}
$$



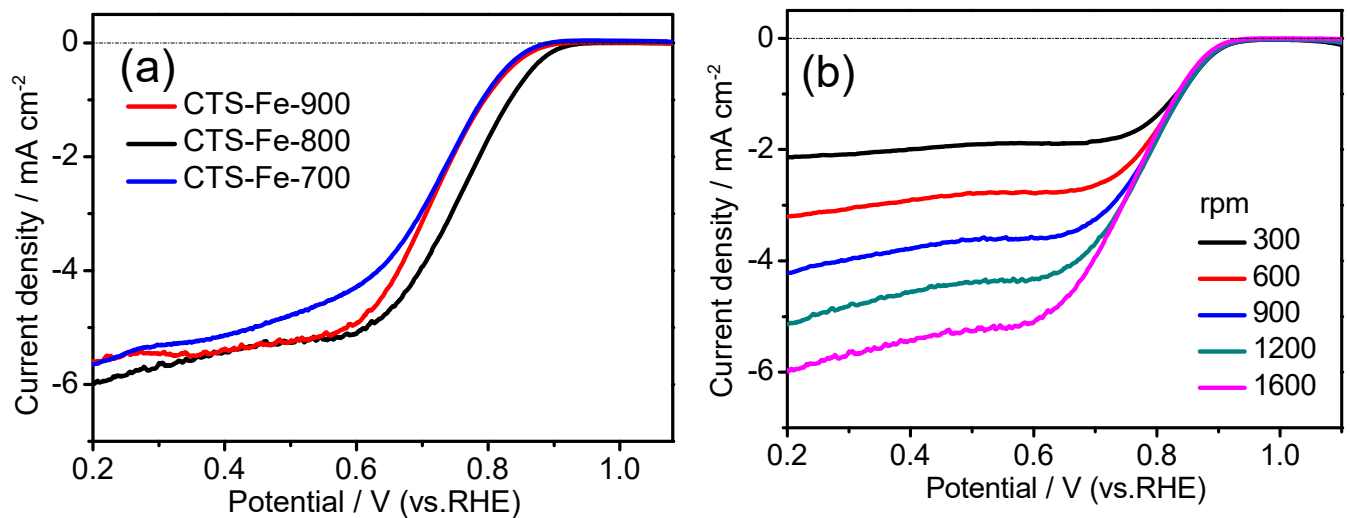

Fig. 2 (a) Polarizationcurves of CTS-Fe-700,-800,-900 in $\mathrm{O}_{2}$-saturated $0.1 \mathrm{M} \mathrm{KOH}$, scan rate: $5 \mathrm{mV} \mathrm{s}^{-1}$, rotation rate: $1600 \mathrm{rpm}$. (b) Polarization curves of CTS-Fe-800 in $\mathrm{O}_{2}$-saturated $0.1 \mathrm{M} \mathrm{KOH}$ at different rotation rates.

Then the $j_{d}$ can be expressed as Eq. 3:

$$
j_{d}=0.2 n F C_{02} D_{02}{ }^{\frac{2}{3}} v^{-\frac{1}{6}} \omega^{\frac{1}{2}}
$$

Where $\mathrm{n}$ is the overall electron transfer number per molecule of $\mathrm{O}_{2}$ reduced, $\omega$ is the electrode rotation rate (rpm), $\mathrm{F}$ is the Faraday's constant $\left(\mathrm{F}=96485 \mathrm{C} \mathrm{mol}^{-1}\right), \mathrm{Co}_{\mathrm{O} 2}$ is the concentration of dissolved oxygen, $\mathrm{Do}_{2}$ is the diffusion coefficient of oxygen in the bulk solution, $v$ is the kinematic viscosity of the solution.

With CTS-Fe-800 as typical candidate, Fig. 3 (a) shows the Koutecky-Levich plots at 0.68, 0.70, 0.74, 0.76, $0.78 \mathrm{~V}$ vs RHE, respectively. By calculating the slopes of the Koutecky-Levich plots, $\mathrm{n}$ value of 3.50, 3.13, 3.58, 3.81 and 3.42 were obtained, respectively. It can be seen that the values of $\mathrm{n}$ for oxygen reduction depend on the high positive over potentials with an average value of 3.49. This indicates that the mixed $2 \mathrm{e}^{-}$and $4 \mathrm{e}^{-}$transfer process took place and, the reduction of $\mathrm{O}_{2}$ produces both $\mathrm{HO}_{2}^{-}$and $\mathrm{OH}^{-}$in the final product on CTS-Fe-800 electrode, but the latter is dominant.

To further investigate the ORR catalytic pathway of the catalyst, rotating ring-disk electrode (RRDE) measurements were also performed to monitor the peroxide yield on the CTS-Fe-800 catalyzed electrode. The peroxide percentage $\left(\mathrm{H}_{2} \mathrm{O}_{2} \%\right)$ was determined from RRDE measurements based on the disk current $\left(I_{\mathrm{d}}\right)$ and ring current $\left(I_{\mathrm{r}}\right)$ via Eq. 4 :

$$
\mathrm{H}_{2} \mathrm{O}_{2} \%=100 \times \frac{2 I_{\mathrm{r}} / N}{I_{d}+I_{r} / N}
$$

The electron transfer number (n) was based on Eq. 5:

$$
n=\frac{4 I_{d}}{I_{d}+I_{r} / N}
$$

Where $N=0.36$ is the current collection efficiency of Pt ring, which is calibrated in $0.1 \mathrm{M} \mathrm{KOH}$ with a $10 \mathrm{mM} \mathrm{K}_{4} \mathrm{Fe}(\mathrm{CN})_{6}$ electrolyte.

As shown in Fig. 3(b), the $\mathrm{H}_{2} \mathrm{O}_{2} \%$ yield on the CTS-Fe-800 electrode is found to be less than $20 \%$ over the potential range of $0 \mathrm{~V}$ to $0.85 \mathrm{~V}$. The corresponding electron transfer number is higher than 3.6, suggesting the nearly complete reduction of oxygen to water. 

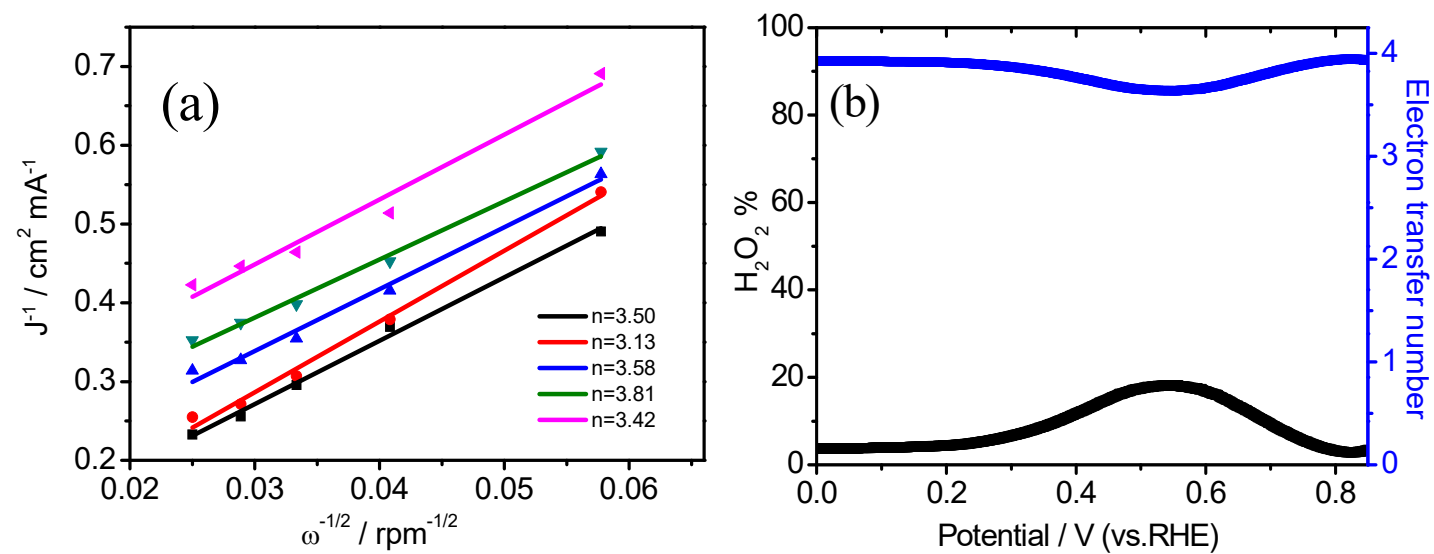

Fig. 3 (a) Koutecky-Levich plots at different electrode potential, which is obtained from $\mathrm{RDE}$ polarization curves of CTS-Fe-800 at different rotation rates for ORR inO $\mathrm{O}_{2}$-saturated $0.1 \mathrm{M} \mathrm{KOH}$ solution. Scan rate: $5 \mathrm{mVs}^{-1}$ and catalyst loading: $80 \mu \mathrm{gcm}^{-2}$. (b) Percentage of $\mathrm{H}_{2} \mathrm{O}_{2}$ produced and the electron transfer number of CTS-Fe-800 (1600 rpm).

\section{Acknowledgements}

This work was financially supported by the National Natural Science Foundation of China (U1510120), the International Academic Cooperation and Exchange Program of Shanghai Science and Technology Committee (14520721900) and the Project of Introducing Overseas Intelligence High Education of China (2016-2017). All the financial supports are gratefully acknowledged.

\section{References}

[1] M.J. Wu, J.L. Qiao, K.X. Li, X.J. Zhou, Y.Y. Liu and J.J. Zhang: Green Chem. Vol. 18 (2016), p. 2699-2709.

[2] M. Arenz, K.J. Mayrhofer, V. Stamenkovic, B.B. Blizanac, T.Tomoyuki, P.N. Ross and N.M. Markovic: J. Am. Chem. Soc. Vol. 127 (2005), p. 6819-6829.

[3] E. Negro, A.H.A.M. Videla, V. Baglio, A.S. Aricò, S. Specchia and G.J.M. Koper: Appl. Catal. B: Environ. Vol. 166-167 (2015), p. 75-83.

[4] C. Xiao, X. Chu, Y. Yang, X. Li, X. Zhang and J. Chen: Biosens. Bioelectron. Vol. 26 (2011), p. 2934-2939.

[5] A.H.A. Monteverde Videla, S. Ban, S. Specchia, L. Zhang and J.J. Zhang: Carbon. Vol. 76 (2014), 386-400.

[6] K. Gong, F. Du, Z. Xia, M. Durstock and L. Dai: J. Sci. Vol. 323 (2009), p. 760-764.

[7] J.L. Qiao, L. Xu, L. Ding, L. Zhang, R. Baker, X. F. Dai and J. J. Zhang: Appl. Catal. B: Environ. Vol. 125 (2012), p. 197-205.

[8] X.F. Dai, J.L. Qiao, J.J. Shi, P.X., L. Zhang and J.J. Zhang: Int. J. Electrochem. Sci., Vol. 8 (2013), p. 3160-3175.

[9] J.J. Shi, M.Y. Fan, J.L. Qiao and Y.Y. Liu: Chem. Lett. Vol. 43 (2014), p. 1484-1486 